\title{
Qualidade da assistência materno-infantil em diferentes modelos de Atenção Primária
}

\author{
Quality of maternal and child health care \\ in different models of Primary H ealth Care
}

Antônio Prates Caldeira ${ }^{1}$

Rafael Morroni de Oliveira ${ }^{1}$

Oreston AlvesRodrigues ${ }^{1}$

'Departamento deSaúdeda Mulher eda Criança, UniversidadeEstadual de Montes Claros. Av. Dr. Rui Braga $s / n^{\circ}$, Vila M auriceia. 39401-347 Montes Claros MG.

antonio.caldera@unimontes.br
Abstract This study evaluated the quality of the maternal and child health care in two different models of Primary H ealth Care. Interviews were carried out by trained personnel with 1200 families randomly selected. Processes of assistance for maternal and child health care were evaluated by Family $\mathrm{H}$ ealth Strategy Teams and traditional health centers. In the evaluation of child health care, the precocity of the first consultation, the regular assessment of growth and development, the recommendations for accident prevention and prophylactic use of iron supplementation and vitamin A had been statistically associated with the model of thehealth care. Regarding prenatal health care the results showed statistically significant differences between the two models for breastfeeding counseling, nutritional recommendationsand cervical preventive screening using Papanicolaou smear. For women health care out of pregnancy period, the results revealed that counseling for breasts auto-examination, preventive screening using Papanicolaou smear in last year and participation in family planning programs were associated with health Primary Health Care model. All the pointed differences had shown better performance of the Family H ealth Strategy Teams. Key words Primary Health Care, M aternal and child health, Family health, O utcome and process assessment (health care)
Resumo Avaliou-se a qualidade da assistência materno-infantil em dois model os de Atenção Primária à Saúde através de entrevistas com 1.200 famílias sel ecionadas aleatoriamente. Foram avaliados indicadores de processo da assistência, comparando a Estratégia de Saúde da Família com centros de saúde tradicionais. $\mathrm{Na}$ avaliação da assistência à criança, a realização precoce da primeira consulta, o acompanhamento do crescimento e desenvolvimento, as orientações para prevenção de acidentes ea profilaxia com sulfato ferroso e vitamina A estiveram estatisticamente associados com o modelo da assistência. $\mathrm{Na}$ assistência pré natal, observou-se diferença estatisticamente significante entre os serviços para orientações sobre amamentação e orientações nutricionais, além da realização de exame de prevenção do câncer de colo uterino. Para os cuidados de saúde da mulher fora do período gestacional, os resultados revelaram que orientações para autoexame das mamas, realização de exame preventivo de câncer de colo uterino no último ano e relato de participação em programa de planejamento familiar estavam associados com o modelo deAtenção Primária à Saúde. Todas as diferenças apontadas mostraram melhor desempenho da Estraté gia de Saúde da Família.

Palavras-chave Atenção Primária à Saúde, Saúde materno-infantil, Saúde da Família, Avaliação de processos e resultados (cuidados de saúde) 
Introdução

No Brasil, a reconstrução do sistema de saúde a partir da implantação do Sistema Ú nico de Saúde procurou, através de seus princípios norteadores, reorientar as práticas de saúde, até então equivocadamente centradas da consulta médica e com destaque para os serviços de emergência ${ }^{1}$. N esse contexto, a implantação da Estratégia de Saúde da Família (ESF) representou um passo fundamental, conciliando um conjunto de ações de promoção, proteção e recuperação da saúde dosindivíduos eda família, desde o recém-nascido ao idoso, de forma integral e contínua. Se gundo os pressupostos da estratégia, a atenção deveestar centrada na família, enten dida e perce bida a partir deseu ambiente físico esocial, o que possibilita às equipes de Saúde da Família uma compreensão ampliada do processo saúde-doença e da necessidade de intervenções que vão além da prática curativa².

Apesar de a especialidade de medicina de família estar se difundindo em todo o mundo, a maioria dos profissionais que trabalha nas equipes não tem especialização na área ou atua há pouco tempo nas atividades da estratégia $a^{3,4}$. Assim, alguns questionamentos são feitos quanto à efetividade da assistência à saúde materno-infantil por profissionais sem uma formação de pósgraduação que os capacite para satisfazer a maior parte das necessidades de saúde que podem apre sentar os pacientes de qualquer idade da comunidade onde trabal ham. 0 primeiro estudo nacional que procurou avaliar qualitativamente o cuidado primário materno-infantil após a implantação da Estratégia de Saúde da Família foi realizado em São Paulo e buscou avaliar a relação entre a utilização de serviços de Atenção Primária e o nível de saúde das crianças ${ }^{5}$. Os resultados desse estudo destacavam que a maioria das crianças tinha acesso a uma atenção fragmentada, com associação direta entre esse tipo de assistência eo seu estado de saúde. Destaca-se, assim, a relevância do cuidado primário continuado, pois acredita-se que a longitudinalidade esteja intimamente associada com a qualidade da atenção ${ }^{6}$.

0 pediatra é, por definição, um médico de cuidado primário $0^{4,7}$. Todavia, em geral, esse profissional tem uma clientela distinta daquela que o médico generalista ou defamília. No entanto, existe uma sobrecarga de demanda para a assistência curativa, particularmente devida a problemas respiratórios e doenças infecciosas ${ }^{8}$, fato que, teoricamente, reduz a eficiência desseprofissional para atendimento preventivo efunções deeducação em saúde. De forma similar, pode-se assumir que o ginecologista-obstetra também efetua atividades prioritárias deA tenção Primária na atenção à gestante e promoção da saúde da mulher.

A ESF, atuando de forma multidisciplinar e prioritariamente com a promoção da saúde, tem, em tese, as melhores condições para promover a saúde infantil e materna, ainda que não tenha em sua composição o profissional especialista. Todavia, a Atenção Primária não se resume a cuidados preventivos, e a continuidade da atenção está muito mais vinculada à responsabilidade profissional do que ao contexto de equipe, conforme aponta M cW hinney? ${ }^{7}$. Se por um lado as medidas elementares desaúde pública representam as primeiras necessidades em muitas sociedades, por outro deve-se reconhecer que não são as únicas. Assim, o questionamento que se faz é se estariam os médicos de família e suas respectivas equipes atentas a tais necessidades, mesmo não dispondo dos ol hares dos especialistas. M otivado por esse hiato de informações, o presente trabalho propôs-se a uma avaliação normativa da ESF em fornecer os cuidados primários à população a ela vinculada, tomando como referencial, ou como marcador, a saúde materno-infantil.

De modo geral, a maioria das propostas avaliativas registradas na literatura referencia os estudos de Donabedian ${ }^{9,10}$. 0 autor propôs que a avaliação da qualidade deveria se dar em três níveis: (1) avaliação da estrutura, que demanda necessariamente a investigação sobre os recursosfísicos, organizacionais e técnicos; (2) avaliação do processo, queimplica a análise dos procedimentos entre servidor e consumidor (não necessariamente a relação médico-paciente, embora muitas vezes seja esta a atividade central do processo); e (3) avaliação de resultado, que discrimina a situação final de saúde (individual ou coletiva) como resultante da complexa interação entre servidor e consumidor das ações de saúde. Classicamente, os estudos que avaliam resultados de ações de saúde são considerados mais consistentes, em uma perspectiva populacional, apesar da dificuldade para a determinação de quais aspectos da saúde devem ser considerados de responsabilidade dos serviços de saúde ${ }^{6}$.

A partir dos pressupostos apresentados, 0 presente estudo teve como objetivo avaliar a qualidade da assistência materno-infantil em áreas assistidas pela ESF estabelecendo uma análise comparativa com os serviços tradicionais deAtenção Primária (centros de saúde) disponíveis para a população, apresentando indicadores de processo vinculados a essa assistência. 


\section{Métodos}

0 presenteestudo foi conduzido em M ontes Claros, ao norte de M inas Gerais. Embora geográfica e politicamente inserido na região Sudeste, 0 norte do estado de M inas Gerais tem indicadores socioeconômicos mais próximos da região N ordeste, mantendo os contrastes emblemáticosdaquilo quese convencionou chamar de"dois Brasis". A cidade sede do estudo possui, atualmente, cerca de 350.000 habitantes erepresenta o principal polo regional. A população é predominantemente urbana (apenas 5,8\% da população se concentra na área rural). As unidades da ESF estão estrategicamentelocalizadas em áreas periféricas da cidadee aten dem as comunidades mais carentes, assumindo uma cobertura de aproximadamente $40 \%$ da população total. A Secretaria M unicipal deSaúdeainda mantém 15 centros de saúde, distribuídos em macrorregiões da cidade, com atendimentos declínica médica, pediatria eginecologia/obstetrícia.

Após a definição das áreas de cobertura da ESF e do modelo tradicional (centros de saúde), foi realizado um inquérito domiciliar com amostra representativa de usuários do cuidado materno-infantil em ambas as áreas. A unidade amostral foi o setor censitário, com exclusão dos setores especiais (domicílios coletivos), das áreas limítrofes (ou apenas parcialmente cobertas pela ESF) e das áreas com equipes de saúde da família incompletasou implantadas há menos deum ano.

Como o tamanho da amostra de domicílios necessários para o estudo é diferente para crianças e mulheres, o cálculo amostral do número de domicílios foi realizado, então, a partir do número de crianças, pois este representava um número maior. 0 tamanho da amostra sugerido por Barros e Victora ${ }^{11}$ é de 450 crianças, e o número fornecido pelo software Epi Info é de 564 crianças. Considerando-se um número ainda maior de crianças para a real ização do inquérito (seiscentas crianças), o número médio de moradores por domicílio na área urbana da cidade (cinco) e a proporção de crianças com idade menor de dois anos de idade (5\%), o número de domicílios a serem visitados seria de aproximadamente 2.400. Como a média de domicílios em cada setor censitário é de 250, um total de dez setores seria suficiente. No presente estudo, duplicou-se esse número de setores censitários, definindo-se que os domicílios seriam visitados de forma alternada, de modo a melhorar qualitativamente a amostra. Em suma, entre todos os setores censitários el egíveis dentro da área assis- tida pela ESF, vinte setores foram al eatoriamente selecionados, o mesmo ocorrendo para os setores censitários incluídos nas áreas assistidas pelos centros de saúde tradicionais.

0 instrumento de coleta de dados foi especialmente desenvolvido para a pesquisa e ajustado após estudo-piloto. Uma equipe de estudantes de medicina eenfermagem da Universidade Estadual de M ontes Claros foi treinada para coleta de dadose codificação das respostas. 0 controledequalidade dos dados foi assegurado com reentrevistas e revisão do processo de codificação em uma amostra al eatória de $5 \%$ do total de entrevistas.

Todas as informações coletadas foram codificadas e digitadas. As análises foram realizadas através do software Epi Info. A associação entre variáveis foi verificada por meio do teste do Quiquadrado, admitindo-se um nível de significância de $5 \%$.

Não foram eletivos para este estudo crianças maiores de dois anos de idade, no sentido de se evitar o viés de memória. Também foram excluídas da avaliação da assistência à saúde feminina as mulheres adolescentes (menores de vinteanos) e as que tinham idade superior a 49 anos, pela possi bilidade de assistência especializada queesses grupos podem demandar. Foram considerados excluídos (ou perdidos) os domicílios sem pessoas habilitadas para responder à entrevista após serem visitados em três dias diferentes, em horários diferentes.

0 presente estudo foi aprovado pelo Comitê de Ética da Unimontes, e todos os entrevistados assinaram termo de consentimento livree esclarecido.

\section{Resultados}

Para avaliação de indicadores de saúde da criança, foram consolidadas 595 entrevistas com mães de crianças menores de dois anos de idade, sendo 297 em áreas assistidas por equipes de saúde da família e 298 em áreas assistidas por centros de saúde tradicionais. Para a avaliação de indicadores de saúde da mulher, foram consolidadas trezentas entrevistas com mulheres residentes em áreas assistidas por equipes de saúde da família e 299 entrevistas com mulheres residentes em áreas de abrangência dos centros desaúdetradicionais.

Apesar do número de entrevistas realizadas, 0 número de informações efetivamente utilizadas variou segundo as características de cada indicador, evitando-se vieses na interpretação dos resultados. Os indicadores vinculados à assistência 
pré-natal representam bem a restrição numérica das informações, pois uma particularidade do município possibilita à gestante realizar 0 acompanhamento pré-natal na unidade de sua preferência (incluindo outras unidades básicas ou maternidades). Assim, para as variáveis relacionadas com a assistência pré natal foram avaliados apenas os dados referentes aos atendimentos efetivamente prestados nas respectivas unidades de saúde. Ou seja, mães residentes em áreas de saúde da família e que foram assistidas nas respectivas unidades foram comparadas com mães residentes em áreas dos centros de saúde e que foram assistidas nos próprios centros de saúde. Para outros indicadores relacionados com a saúde da mulher, foram computadas apenas as informações referentes às mulheres com idade entre 20 e 49 anos, conforme definido previamente.

As características socioeconômicas dos grupos estudados foram avaliadas apenas para as famílias cuja investigação incluía indicadores de saúde infantil e são apresentadas na Tabela 1.

A Tabela 2 apresenta os principais indicadores de processo da assistência à saúde da criança investigados no estudo, e a Tabela 3 apresenta os principais indicadores de processo da assistência à saúde da mulher.

É importante salientar dois indicadores que não estão presentes nas tabelas apresentadas: a proporção de crianças com baixo peso ao nascer e a proporção de partos cesáreas. Ambos os indicadores podem ser tomados como indicadores de processo ou de resultado. Contudo, fo- ram excluídos das tabelas pela possibilidade de múltiplas interferências não contempladas no estudo. A proporção de recém-nascidos de baixo peso foi de $10,4 \%$ nas áreas assistidas pela ESF e de $8,4 \%$ nasáreas assistidas pelos centros de saúde tradicionais (não se observando diferença estatisticamente significante entre as proporções). Para a proporção de partos cesáreas, os números observados foram de $21,7 \%$ e $29,5 \%$, respectivamente, nas áreas da ESF e nas áreas de assistência tradicional (diferença também não significante, estatisticamente).

\section{Discussão}

0 presente estudo apresenta uma abordagem de avaliação da Atenção Primária na perspectiva da avaliação normativa de processo da assistência, cujos resultados apontam melhor desempenho das atividades desenvolvidas pelas equipes desaúde da família, em relação aos aspectos investigados. A generalização dos dados deve ser tomada com cautela, pois existem al gumas limitações do processo de investigação. Trata-se de um estudo restrito a um único município, com particularidades próprias de sua posição geográfica. Salienta-se ainda que, embora a área de abrangência tenha sido limitada àquel as com equipes completas constituídas há pelo menos um ano, o tempo deexistência da equipenão foi tomado como uma variável na análise de dados, sendo essa questão importante no processo de adesão dos pacientes

Tabela 1. Variáveis socioeconômicas, segundo o modelo de Atenção Primária à Saúde. M ontes Claros (M G), 2006.

\begin{tabular}{|c|c|c|c|c|c|}
\hline \multirow{2}{*}{ Característica } & \multicolumn{2}{|c|}{ ESF $^{*}$} & \multicolumn{2}{|c|}{$\mathrm{CS}^{* *}$} & \multirow{2}{*}{ OR (IC95\%) } \\
\hline & (n) & $(\%)$ & $(n)$ & $(\%)$ & \\
\hline \multicolumn{6}{|l|}{ Escolaridade mãe } \\
\hline$<4$ anos & 43 & 14,5 & 16 & 5,4 & $3,55(1,86-6,86)$ \\
\hline 4-8 anos & 96 & 32,3 & 73 & 24,5 & $1,74(1,18-2,56)$ \\
\hline$>8$ anos & 158 & 53,2 & 209 & 70,1 & 1,00 \\
\hline \multicolumn{6}{|l|}{ Pai na estrutura familiar } \\
\hline Não & 67 & 22,6 & 65 & 21,8 & $1,04(0,70-1,57)$ \\
\hline Sim & 230 & 77,4 & 233 & 78,2 & 1,00 \\
\hline \multicolumn{6}{|l|}{ H abitantes por domicílio } \\
\hline$>5$ habitantes & 149 & 50,2 & 142 & 47,7 & $1,11(0,79-1,55)$ \\
\hline$<5$ habitantes & 148 & 49,8 & 156 & 52,3 & 1,00 \\
\hline \multicolumn{6}{|c|}{ Família beneficiária de programa social } \\
\hline Sim & 139 & 46,8 & 102 & 34,2 & $1,69(1,20-2,39)$ \\
\hline Não & 158 & 53,2 & 196 & 65,8 & 1,00 \\
\hline
\end{tabular}

"ESF: Estratégia de Saúde da Família; ${ }^{*}$ CS: Centro de saúde. 
Tabela 2. Indicadores de processo de assistência à saúde da criança, segundo o modelo de Atenção Primária à Saúde. M ontes Claros (M G), 2006.

\begin{tabular}{|c|c|c|c|c|c|c|}
\hline \multirow[t]{2}{*}{ Característica } & \multicolumn{2}{|c|}{$\mathrm{ESF}^{*}$} & \multicolumn{2}{|c|}{$\mathrm{CS}^{* *}$} & \multirow[t]{2}{*}{ “p” } & \multirow[t]{2}{*}{ OR (IC95\%) } \\
\hline & $\mathrm{N}$ & $\%$ & $\mathrm{~N}$ & $\%$ & & \\
\hline \multicolumn{7}{|l|}{ Controle regular/puericultura } \\
\hline $\operatorname{Sim}$ & 225 & 75,8 & 176 & 59,1 & & 1,0 \\
\hline Não & 72 & 24,2 & 122 & 40,9 & 0,000 & $2,17(1,50-3,13)$ \\
\hline \multicolumn{7}{|l|}{ Realização primeira consulta } \\
\hline$\leq 7$ dias & 73 & 24,6 & 32 & 10,7 & & 1,00 \\
\hline $8-15$ dias & 120 & 40,4 & 96 & 32,2 & 0,023 & $1,83(1,08-3,09)$ \\
\hline$>15$ dias & 104 & 35,0 & 170 & 57,1 & 0,000 & $3,73(2,24-6,22)$ \\
\hline \multicolumn{7}{|l|}{ Registro regular do "CD" } \\
\hline Sim & 216 & 72,7 & 144 & 48,3 & & 1,00 \\
\hline Não & 81 & 27,3 & 154 & 51,7 & 0,000 & $2,85(2,00-4,07)$ \\
\hline \multicolumn{7}{|l|}{ Cartão vacinal em dia } \\
\hline Sim & 289 & 97,3 & 284 & 95,3 & & 1,00 \\
\hline Não & 08 & 2,7 & 14 & 4,7 & 0,281 & $1,78(0,69-4,71)$ \\
\hline \multicolumn{7}{|l|}{ U so profilático de FeSO 4} \\
\hline Sim & 117 & 57,4 & 95 & 46,6 & & 1,00 \\
\hline Não & 87 & 42,6 & 109 & 53,4 & 0,037 & $1,54(1,02-2,33)$ \\
\hline \multicolumn{7}{|l|}{ U so regular de vitamina $A$} \\
\hline $\operatorname{Sim}$ & 186 & 86,9 & 144 & 70,2 & & 1,00 \\
\hline Não & 28 & 13,1 & 61 & 29,8 & 0,000 & $2,81(1,66-4,77)$ \\
\hline \multicolumn{7}{|c|}{ Orientações prevenção de acidentes } \\
\hline Sim & 88 & 29,7 & 54 & 18,1 & & 1,00 \\
\hline Não & 209 & 70,4 & 244 & 84,9 & 0,001 & $1,90(1,27-2,85)$ \\
\hline
\end{tabular}

*ESF: Estratégia de Saúde da Família; ${ }^{*}$ CS: Centro de saúde.

às recomendações de saúde. Outro aspecto que também pode interferir na interpretação dos dados diz respeito à capacitação dos recursos humanos agregados às unidades de cuidado primário, variável também não inserida na presenteavaliação. Contudo, os resultados do presente estudo podem ser tomados em conjunto com outras pesquisas, que têm mostrado uma interferência positiva da ESF, especialmente vinculada à população mais necessitada ${ }^{12,13}$.

A revisão da literatura destaca que os pressupostos mais utilizados pelos estudos de avaliação da qualidade da assistência têm como referência as investigações de processos e resultados $^{14}$. 0 presente estudo procurou, nessa perspectiva, propiciar uma avaliação externa deindicadores de processo da Atenção Primária. Embora exista uma proposta explícita do M inistério da Saúde de grandes investimentos na área ${ }^{15}$, contam-se poucos estudos avaliativos semelhantes ${ }^{5,12,16}$. A avaliação da Estratégia de Saúde da Família, em particular, representa uma importante ferramenta para eventuais ajustes do projeto, apresentado em meados da década passada como uma proposta de reformulação do modelo assistencial.

A complexidade do tema e a subjetividade, quase sempre inerente ao processo avaliativo, tornam o objeto "qualidade da assistência" demasiado amplo e de múltiplos caminhos metodológicos. Ou seja, além de os estudos avaliativos em Atenção Primária não se apresentarem com o merecido destaque na literatura, os diferentes recortes metodológicos tornam mais difícil a análise comparativa dos seus resultados.

A Tabela 1 mostra que a população adscrita às unidades da ESF é significativamente menos favorecida, apresentando menores índices de escolaridade, um indicador indireto de renda e acesso a benseserviçosem geral. Outro indicador decarência social apresentado na tabela éo fato detambém se incluir nessa população maior proporção defamílias com crianças beneficiárias de programas sociais (bolsa-família, cesta básica etc.). Os dados observados estão em consonância com a proposta daESF. Embora não seja um processo excludente, em sua faseinicial, atendendo ao princípio da equidade, a ESF se destina, prioritariamente, às cama- 
Tabela 3. Indicadores de processo de assistência à saúde da mulher, segundo o modelo de Atenção Primária à Saúde. M ontes Claros (M G), 2006.

\begin{tabular}{|c|c|c|c|c|c|c|}
\hline \multirow[t]{2}{*}{ Característica } & \multicolumn{2}{|c|}{$\mathrm{ESF}^{*}$} & \multicolumn{2}{|c|}{$\mathrm{CS}^{* *}$} & \multirow[t]{2}{*}{ "p" } & \multirow[t]{2}{*}{ OR (IC95\%) } \\
\hline & $\mathrm{N}$ & $\%$ & $\mathrm{~N}$ & $\%$ & & \\
\hline \multicolumn{7}{|l|}{ Consultas pré-natais } \\
\hline$\geq 6$ consultas & 90 & 78,3 & 122 & 73,5 & & 1,0 \\
\hline$<6$ consultas & 25 & 21,7 & 44 & 26,5 & 0,440 & $1,30(0,71-2,37)$ \\
\hline \multicolumn{7}{|l|}{ Início do pré-natal } \\
\hline 10 trimestre & 80 & 69,6 & 110 & 66,3 & & 1,0 \\
\hline 20 ou 3o trimestre & 35 & 30,4 & 56 & 33,7 & 0,652 & $1,16(0,68-2,00)$ \\
\hline \multicolumn{7}{|l|}{ Orientações sobre AM no PN } \\
\hline Sim & 106 & 92,2 & 138 & 83,1 & & 1,0 \\
\hline Não & 9 & 7,8 & 28 & 16,9 & 0,043 & $2,39(1,02-5,72)$ \\
\hline \multicolumn{7}{|l|}{ Orientação nutricional gestante } \\
\hline Sim & 97 & 84,3 & 118 & 71,1 & & 1,0 \\
\hline Não & 18 & 15,7 & 48 & 28,9 & 0,015 & $2,19(1,15-4,20)$ \\
\hline \multicolumn{7}{|l|}{ Realização de PCCU no PN } \\
\hline Sim & 50 & 43,5 & 35 & 21,1 & & 1,0 \\
\hline Não & 65 & 56,5 & 131 & 78,9 & 0,000 & $2,88(1,65-5,04)$ \\
\hline \multicolumn{7}{|l|}{ Uso de ferruginoso no PN } \\
\hline Sim & 91 & 79,1 & 122 & 73,5 & & 1,0 \\
\hline Não & 24 & 20,9 & 44 & 26,5 & 0,346 & $1,37(0,75-2,51)$ \\
\hline \multicolumn{7}{|l|}{ Avaliação odontológica no PN } \\
\hline Sim & 14 & 12,2 & 13 & 7,8 & & 1,0 \\
\hline Não & 101 & 87,8 & 153 & 92,2 & 0,313 & $1,63(0,69-3,87)$ \\
\hline \multicolumn{7}{|l|}{ Cartão de PN completo } \\
\hline Sim & 111 & 96,5 & 154 & 92,8 & & 1,0 \\
\hline Não & 04 & 3,5 & 12 & 7,2 & 0,284 & $2,16(0,63-8,18)$ \\
\hline \multicolumn{7}{|l|}{ Orienta autoexame (mamas) } \\
\hline Sim & 187 & 63,0 & 160 & 53,5 & & 1,0 \\
\hline Não & 110 & 37,0 & 139 & 46,5 & 0,024 & $1,48(1,05-2,08)$ \\
\hline \multicolumn{7}{|l|}{ Última consulta ginecológica } \\
\hline$\leq 12$ meses & 205 & 68,3 & 213 & 71,2 & & 1,0 \\
\hline$>12$ meses & 95 & 31,7 & 86 & 28,8 & 0,493 & $0,87(0,61-1,25)$ \\
\hline \multicolumn{7}{|l|}{ Último exame de PCCU } \\
\hline$\leq 12$ meses & 190 & 63,3 & 162 & 54,2 & & 1,0 \\
\hline$>12$ meses & 110 & 36,7 & 137 & 45,8 & 0,028 & $1,46(1,04-2,05)$ \\
\hline \multicolumn{7}{|l|}{ Último exame das mamas } \\
\hline$\leq 12$ meses & 133 & 44,6 & 130 & 43,6 & & 1,0 \\
\hline$>12$ meses & 165 & 55,4 & 168 & 56,4 & 0,869 & $1,04(0,74-1,46)$ \\
\hline \multicolumn{7}{|l|}{ Planejamento familiar } \\
\hline Sim & 149 & 50,0 & 122 & 41,4 & & 1,0 \\
\hline Não & 149 & 50,0 & 173 & 58,6 & 0,032 & $1,44(1,03-2,02)$ \\
\hline
\end{tabular}

*ESF: Estratégia de Saúde da Família; ${ }^{* *}$ CS: Centro de saúde.

das menos favorecidas da população. Esses achados não implicam distorções do processo de seleção, quefoi realizado deforma absolutamente aleatória dentro de cada área assistida pelos diferentes modelos. Resultados semelhantes foram apontados em outros estudos ${ }^{12,13}$.

A Tabela 2 mostra os indicadores de processo da assistência à saúde da criança, destacando queo controle regular de puericultura, a realização pre coce da primeira consulta, o registro regular do acompanhamento do crescimento e do desenvolvimento ("CD"), as orientações para prevenção de acidentes na infância eo uso profilático do sulfato ferroso e da vitamina A são atividades que estão estatisticamente associadas com a adscrição da família da criança em área da ESF $(p<0,05)$. 
Embora seja eventualmentedifícil estabelecer parâmetros de bom desempenho para as atividades de assistência à saúde da criança, existem suficientes evidências na literatura que dão suporte às variáveis estudadas. Assim, o controle regular de puericultura está associado ao diagnóstico precoce e intervenções oportunas e positivas para a saúde da criança ${ }^{17}$. De forma similar, a primeira consulta realizada de forma mais pre coce pode antecipar interven ções oportunas, permitindo identificar fatores de risco para o desmame precoce e corrigindo distorções dos cuidados iniciais com a criança ${ }^{17}$. Também existem evidências que destacam o valor de orientações específicas para preven ção deacidentes na infân$c i a^{18}$. O uso profilático do sulfato ferroso e da vitamina A também são estratégias recomendadas pelo M inistério da Saúde (no caso da vitamina $A$, apenas para al gumasáreas de risco em todo o país) com potencial de evitar agravos nutricionais importante ${ }^{19,20}$. Um grande estudo realizado por Fachini e colaboradores (dentro do projeto de avaliação de linha de base de expansão da Estratégia deSaúde da Família), em análisecomparativa entre a ESF e o modelo tradicional em 41 municípios nas regiões Nordeste e Sul, também mostrou maior proporção de crianças em acompanhamento de puericultura em áreas assistidas por equipes de saúde da família12.

Também está em consonância com os resultados apresentados o fato de o referido estudo ter apontado melhor desempenho das unidades de saúde da família para outras atividades de cuidados com a criança (manejo dos agravos infantis mais prevalentes, manejo da desnutrição, promoção do aleitamento materno e visita domiciliar).

Os cuidados de promoção da saúde da criança são relativamente simples, mas demandam tempo e dedicação por parte do profissional de saúde. Diante dos resultados apresentados, é natural concluir que as atividades dos centros de saúde tradicionais em relação à saúde da criança estão ainda vinculadas ao modelo biomédico ${ }^{21}$. Assim, ações de promoção e prevenção estão, quase sempre, desvinculadas das ações curativas, e prevalecem os atendimentos fragmentados e pontuais.

A Tabela 3 mostra os indicadores de processo da assistência à saúde da mulher. Especificamente em relação ao atendimento pré-natal, foram verificadas diferen ças estatisticamentesignificantes entre os dois modelos de assistência para as seguintes variáveis: orientações sobre aleitamento materno durante o pré-natal, orientações nutricionais para a gestante e realização de exa- me de prevenção do câncer de colo uterino (PCCU) durante o pré-natal. Em relação aos cuidados de saúde da mulher fora do período gestacional, mostraram-se associadas com 0 modelo de atenção: orientações para o autoexame das mamas, realização de exame preventivo de câncer de colo uterino nos últimos 12 mesese relato de participação ou inscrição em programa de planejamento familiar. Todas as diferenças apontadas foram em favor da ESF. Salienta-se que as variáveis estudadas foram selecionadas a partir do rol de atividades recomendadas em relatórios técnicos do M inistério da Saúde.

Os resultados apresentados em relação à saúde da mulher também estão em consonância com os resultados comparativos de Facchini e colaboradores para municípios da região Sul do Brasil ${ }^{12}$. Outro estudo queavaliou os critérios deescolha de local para atendimento pré natal também identificou que as atividades dos centros de saúde são menos efetivas do que outros serviços na prescrição do sulfato ferroso para as gestantes, na completude do exameginecológico, no examedas mamas e nas orientações para amamentação ${ }^{22}$.

É importante apontar que, embora as recomendações para realização anual do exame de prevenção de câncer de colo uterino (PCCU) e para o autoexame das mamas sejam variáveis passíveis de crítica, por não mostrarem significativa efetividade, ainda representam um importante de indicador de acesso e qualidade da assistência ${ }^{23}$.

Os resultados fornecem subsídios importantes ao sistema deAtenção Primária no país, especialmente quando associados a outros estudos. Há indícios de que o processo da assistência à saúde materno-infantil é mais adequado no modelo da ESF em vários aspectos. Esse resultado representa um aspecto fundamental da pesquisa, pois apresenta um dado real decorrente da proposta político-ideológica concebida através da ESF. Um dos possíveis recortes da pesquisa de avaliação assegura que um determinado serviço é de boa qualidade quando o processo de assistência apresenta certas características que a experiência (ou estudos preliminares) associa com resultados desejávei $s^{24}$. Ou seja, embora não exista nenhuma garantia de que os processos de assistência assegurem resultados positivos para a saúde da população, existe a premissa de que, em estudos avaliativos, bons processos de assistência à saúde levam a bons resultados ${ }^{25}$.

Deve-se destacar a dificuldade de trabalhar com indicadores de resultados em análises comparativas para o atual estágio da ESF. 0 estudo de Roncalli e Lima ${ }^{16}$, por exemplo, não conse- 
guiu aferir diferenças significativas em entre indicadores de resultado comparando-se equipes de saúde da família e outros serviços. Como ainda está em fase de implantação/expansão para a maior parte do país, a estratégia carece de uniformidade em um dos seus pressupostos bási$\cos , 0$ da longitudinalidade. Para Starfield, a longitudinalidade é uma das premissas para avaliação deimpacto ou resultados em serviços deAtenção Primária 6 .

Registra-se ainda que os processos de trabaIho estão inseridos em áreas e populações mais carentes (Tabela 1) e que essas ações mostram a possibilidade de se amenizarem os efeitos da pobreza sobre a saúde da população adscrita. Ou seja, a estratégiatem representado um esforço bemsucedido de aumentar 0 acesso e a equidade para as camadas menos favorecidas da população.

$N$ ão se avaliou no presente estudo a efetividade dos serviços para a assistência de problemas específicos de saúde, o que não representa uma ação prioritariamente vinculada ao cuidado primário. 0 país atravessa uma fase de rápida transição epidemiológica, em queas doenças agudase infecciosas têm cada vez menosinfluência na definição do perfil nosológico da população, en quanto as doenças crônicas e degenerativas ocupam cada vez maior destaque nos indicadores de morbimortalidade. Não é sensato, nesse momento, perseverar na lógica do atendimento às condições agudas, que negligencia as práticas educativas, de prevenção e de promoção da saúde, especialmente em relação à saúde materno-infantil.

A coexistência de dois modelos distintos parece ser uma realidade para a maioria das cidades de grande e médio portes no Brasil ${ }^{12,13,16}$. Diante das conclusões naturais de que a assistência à saúde da criança e da mulher nos centros de saúde se mostra, quase sempre, fragmentada e respondendo apenas às demandas pontuais da população, caberá aos gestores de saúde a tarefa de romper com a estrutura já consolidada dos centros de saúde tradicionais, agregando ao rol de suas atividades ações sistemáticas de promoção da saúde ou estimulando a ampliação da Estratégia de Saúde da Família.

Concluindo, é importante destacar que, embora tenham apresentado melhor desempenho, as unidades de saúde da família ainda estão aquém das necessidades da população. Muitas ações devem ser melhoradas, e novas ações são ainda necessárias para aumentar a efetividade das equipes. De forma análoga, novos estudos são ainda necessários para continuar avaliando que impacto a reorganização do sistema de saúdetem apresentado sobre o status de saúde e sobre 0 acesso ao cuidado de saúde para as mães e as crianças brasileiras.

\section{Colaboradores}

AP Caldeira participou na concepção do projeto, no delineamento do trabalho de campo, na análise e interpretação dos dados, na redação e análise crítica do texto final. RM Oliveira e OA Rodrigues participaram da coleta dos dados, análise e interpretação dos dados e redação do trabaIho final.

\section{Agradecimentos}

Os autores agradecem o financiamento do CN Pq, sem o qual este estudo não teria sido possível. 


\section{Referências}

1. Mendes EV. Uma agenda para a saúde. São Paulo: Hucitec; 1996.

2. Ribeiro EM. As várias abordagens da família no cenário do Programa/Estratégia de Saúde da Família (PSF). Rev Latino Am Enfermagem 2004; 12(4):658664.

3. Brasil. M inistério da Saúde. Secretaria de Atenção à Saúde. Avaliação normativa do Programa Saúde da Família no Brasil: monitoramento da implantação e funcionamento das equipes de saúde da família: 20012002. Brasília: Ministério da Saúde; 2004.

4. Haq C, Ventres W, Hunt V, Mull D, Thompson R, Rivo $M$, Johnson $P$. Where there is no family doctor: the development of family practice around the world. Acad M ed 1995; 70(5):370-380.

5. Moura EC. The relationship between the use of primary care and infant health status at 12 months in Brazilian community. Rev Bras Epidemiol 1998; 1(1):79-87.

6. Starfield B. Atenção Primária: equilíbrio entre necessidades de saúde, serviços e tecnologia. Brasília: Unesco, M inistério da Saúde; 2004.

7. M cWhinney IR. M edicina de família. Barcelona: M osby/Doyma Libros; 1995.

8. Durá Travé T, Gúrpide Ayarra N. Presión asistencial, frecuentación y morbilidad pediátrica en un centro de salud: diferencias etarias y estacionales. Aten Primaria 2001; 27(4):244-249.

9. Donabedian A. Evaluating the quality of medical care. M ilbank M em Fund Q 1966; 44(3):166-206.

10. Donabedian A. Promoting quality through evaluating the process of patient care. Med Care 1968; 6(3):181-202.

11. Barros FC, Victora CG. Epidemiologia da saúde infantil: um manual para diagnósticos comunitários. São Paulo: Hucitec, Unicef; 1998.

12. Facchini LA, Piccini RX, Tomasi E, Thumé E, Silveira DS, Siqueira FV, Rodrigues MA. Desempenho do PSF no Sul e no Nordeste do Brasil: avaliação institucional e epidemiológica da atenção básica à saúde. Cien Saude Colet 2006; 11(3):669-681.

13. Goldbaum M, Gianini RJ, Novaes HMD, César CLG. Utilização de serviços de saúde em áreas cobertas pelo programa saúde da família (Qualis) no município de São Paulo. Rev Saude Publica 2005; 39(1):90-99.

14. Reis EJFB, Santos FP, Campos FE, Acúrcio FA, Leite MTT, Leite M LC, Cherchiglia ML, Santos MA. Avaliação da qualidade dos serviços de saúde: notas bibliográficas. Cad Saude Publica 1990; 6(1):50-61.
15. Felisberto E. M onitoramento e avaliação na atenção básica: novos horizontes. Rev Bras Saúde Mater Infant 2004; 4(3):317-321.

16. Roncalli AG, Lima KC. Impacto de programas de saúde da família sobre indicadores de saúde da criança em municípios de grande porte da região Nordeste do Brasil. Cien Saude Colet 2006; 11(3):713-724.

17. Needlman RD. Growth and development. In: Behrman RE, Kliegman RM, Harbin AM. N elson textbook of pediatrics. Philadelphia: WB Saunders Company; 1996. p. 30-72.

18. Rivara FP. Prevention of injuries to children and adolescents. Inj Prev 2002; 8(Suppl.IV):5-8.

19. Abreu J, Borno S, Montilla M, Dini E. Anemia y deficiencia de vitamina $A$ en niños evaluados en un centro de atención nutricional de Caracas. Arch Latinoam Nutr 2005; 55(3):226-234.

20. Villalpando $S$, Pérez-Expósito AB, Shamah-Levy $T$, Rivera JA. Distribution of anemia associated with micronutrient deficiencies other than iron in a probabilistic sample of Mexican children. Ann Nutr M etab 2006; 50(6):506-511.

21. M cElmurry BJ, Marks BA, Cianelli R. Primary health care in the Americas: conceptual framework, experiences, challenges and perspectives. Washington, D.C.: Pan American Health Organization; 2002.

22. Santos IS, Baroni RC, M inotto I, Klumb AG. Critérios de escolha de postos de saúde para acompanhamento pré-natal em Pelotas, RS. Rev Saude Publica 2000; 6(34):603-609.

23. Swenson DE. Pap smear screening during pregnancy. Necessary component of the first prenatal visit. Adv Nurse Pract 2001; 9(8):53-56.

24. M yers RG. Notas sobre la calidad de la atención a la infância. Rev Latinoam Cienc Soc Niñez-Juv 2003; 1(1):59-83.

25. Campos CEA. Estratégias de avaliação e melhoria contínua da qualidade no contexto da Atenção Primária à Saúde. Rev Bras Saúde M ater Infant 2005; 5(Supl.1):s63-s69.

Artigo apresentado em 21/05/2007

Aprovado em 11/10/2007

Versão final apresentada em 14/12/2007 